\title{
VARIAÇÃO INTRAESPECÍFICA DOS NUTRIENTES EM FOLHAS DE METRODOREA PUBESCENS (RUTACEAE) EM DOIS TIPOS DE MATA DO DISTRITO FEDERAL.
}

\author{
Marcelo Trindade Nascimento ${ }^{1}$ \\ John Duvall Hay ${ }^{2}$
}

\begin{abstract}
RESUMO - Metrodorea pubescens é uma árvore comum nas matas do Distrito Federal, ocorrendo em matas mesofíticas (solos calcáreos) e matas de galeria (solos ácidos). Em cada tipo de mata foram coletadas todas as folhas de 15 indivíduos jovens, sendo cinco por classe de altura $(5-50,50-100$, e $100-150 \mathrm{~cm})$. As folhas foram separadas em três categorias: novas, maduras e senescentes e depois foram secas e analisados para os seguintes elementos: $\mathrm{P}, \mathrm{K}, \mathrm{Ca}, \mathrm{Mg}$ e $\mathrm{Al}$. A concentração dos nutrientes nas folhas não diferiu em relação ao tamanho dos indivíduos. Apenas o alumínio apresentou menores teores nos indivíduos de $5-50 \mathrm{~cm}$. As folhas novas apresentaram maiores teores de $\mathrm{P}$ e $\mathrm{K}$. Já o $\mathrm{Ca}, \mathrm{Mg}$, e $\mathrm{Al}$ foram maiores nas folhas maduras. Há um decréscimo de $\mathrm{P}$ da folha madura para folha senescente. As plantas do solo calcáreo apresentaram maiores teores de $\mathrm{Ca}$, menores de $\mathrm{K}$, e valores semelhantes de $\mathrm{P}, \mathrm{Mg}$, e $\mathrm{Al}$, quando comparadas com plantas do solo ácido. As diferenças entre as populações talvez possam ser explicadas pelo solo, porém outros fatores como o comportamento fisiológico das plantas devem ser considerados. As plantas do solo ácido podem ter um comportamento calcífugo e as plantas do solo calcáreo um comportamento calcícolo.
\end{abstract}

Palavras-Chave: Metrodorea pubescens, nutrientes foliares, floresta de galeria, floresta mesofítica

ABSTRACT - Metrodorea pubescens is an abundant tree in the forests of the Distrito Federal, occurring in mesophytic forests (on calcareous soils) and in gallery forests (acidic soils). In both forest types all leaves from 15 small individuals from five individuals in each of three height classes $(5-50,50-100,100-150 \mathrm{~cm})$ were collected.

The leaves were separated in three categories; new, mature and senescent, and then dried and analyzed for the following elements: $\mathrm{P}, \mathrm{K}, \mathrm{Ca}, \mathrm{Mg}$ and $\mathrm{Al}$. The concetration of nutrientes in the leaves was sirnilar in all height classes, except for al which showed lower concentration in the smallest individuals $(5-50 \mathrm{~cm})$. New leaves showed higher concentration of $\mathrm{P}$ and $\mathrm{K}$. On the other hand $\mathrm{Ca}, \mathrm{Mg}$ and $\mathrm{Al}$ were higher in the mature leaves. The concentration of $\mathrm{P}$ decreased from mature leaves to senescent leaves. The plants from calcareous soil showed higher concentration of $\mathrm{Ca}$ and lower concentration of $\mathrm{K}$ than plants of acidic soil. The

1 - Aluno do Mestrado em Ecologia da UnB. Bolsista do CNPq.

2 - Professor Adjunto, Depto. de Ecologia, CP 153.081, UnB, Brasília-DF, 70919. 
values of $\mathrm{P}, \mathrm{Mg}$ and $\mathrm{Al}$ were similar in both forests. The differences between populations could possibly be explained by soil type, but other factors such as the physiological behavior of plants should also be considered. Plants from the acidic soil can be calcifuge and plants from the calcareous soil can be calcicole.

Key Words: Metrodorea pubescens, leaf nutrients, gallery forest, mesophytic forest.

\section{Introdução}

Metrodorea pubescens St. Hill. \& Tull. (Rutaceae) é uma espécie comum nas matas do Distrito Federal, ocorrendo tanto em matas mesofíticas (solos calcáreos), quanto em matas de galeria (solos ácidos). O padrão de distribuição espacial desta espécie é agregado, com suas populações, aparentemente, bem estabelecidas nestes dois tipos de mata (Nascimento, 1989).

A ocorrência de uma mesma espécie em locais com condições edáficas tão diversas já tem sido observada por vários autores (Davies e Snaydon 1973, Haridasan e Araújo 1988). Aspectos relacionados à plasticidade fisiológica do indivíduo ou com o polimorfismo fisiológico destas espécies compostas de diferentes populações, cada uma com adaptações a estreitas condições de solo, têm sido os argumentos utilizados para se tentar explicar este fato (Wacquant $\mathrm{e}$ Bouab 1983).

O objetivo deste trabalho foi verificar a ocorrência ou não de diferenças na concentração dos macronutrientes nas folhas de $M$. pubescens de duas populações, uma de solo calcáreo e outra de solo ácido.

\section{Materiais e Métodos}

Áreas de Estudo

O presente trabalho foi desenvolvido no Distrito Federal em dois tipos de mata, mata de galeria e mata mesofítica semidecídua. A mata de galeria estudada está localizada no ribeirão do Gama, na Fazenda Água Limpa (FAL) de propriedade da Universidade de Brasília (470 $\left.57^{\prime} \mathrm{W}, 15^{\circ} 57^{\prime} \mathrm{S}\right)$ cerca de 20 $\mathrm{km}$ do centro de Brasília. Esta mata possui área de aproximadamente 54 hectares, com solo ácido, periodicamente inundado próximo as margens. As espécies arbóreas mais comuns são: Aspidosperma sp., Astronium fraxinifolium, Copaifera langsdorfii, Metrodorea pubescens, e Xylopia emarginata (Felfili, dados não publicados). A localização da mata mesofítica semidecídua estudada está na parte centro-norte do Distrito Federal (470 $52^{\prime} \mathrm{W}, 15^{\circ} 35^{\prime} \mathrm{S}$ ), próximo a cidade de Sobradinho, na rodovia DF-9, junto ao entroncamento com a rodovia DF-2, distando aproximadamente $30 \mathrm{~km}$ do centro de Brasília. Esta mata apresenta uma área de cerca de 30 hectares e cresce sobre solo calcáreo. $\mathrm{O}$ solo é alcalino e apresenta grandes quantidades de bases e fósforo. Em ge- 
ral, os solos de calcáreo das matas mesofíticas do Distrito Federal possuem níveis mais altos de cátions trocáveis, nitrogênio total e fósforo disponível do que os solos das matas de galeria (Haridasan 1985). As espécies arbóreas mais comuns nesta mata mesofítica são Eugenia florida, Metrodorea pubescens, Piptadenia inaequalis, Aspidosperma sp. e Alibertia sp. (Ramos 1989).

A região do Distrito Federal possui um clima correspondente ao tipo Awi de Koppen (Eiten 1984), isto é, tropical com uma estação chuvosa (novembro a abril) e uma estação seca (maio a outubro). A diferença entre as médias de temperatura dos meses mais frios e mais quentes é menor que $5^{\circ} \mathrm{C}$.

\section{Coletas e análises químicas das folhas}

Em cada tipo de mata foram coletadas todas as folhas de 15 indivíduos jovens, sendo cinco por classe de altura $(5-50,50-100$ e $100-150 \mathrm{~cm})$ em dois períodos de amostragem, o primeiro durante o final das chuvas (abril/88) e o segundo durante o final da seca (setembro/88).

As folhas coletadas de cada indivíduo foram separadas em três categorias de idade: folhas novas, maduras e senescentes, baseado na textura, coloração e posicionamento das folhas no ramo. As folhas novas apresentavam textura membranácea, coloração verde clara e estavam localizadas nos ápices dos ramos. As folhas maduras tinham textura mais dura, coloração verde escura e, em geral, estavam localizadas no ramo logo abaixo das folhas novas. As senescentes apresentavam cor verde escura, com manchas amareladas. Seu posicionamento no ramo da planta era abaixo das folhas maduras.

Para as análises químicas dos teores de $\mathrm{P}, \mathrm{K}, \mathrm{Ca}, \mathrm{Mg}$, e $\mathrm{Al}$ as folhas novas, maduras e senescentes de cada indivíduo foram lavadas em água destilada e secas em estufa a $80^{\circ} \mathrm{C}$ por 24 horas e moídas em um moinho do tipo Wiley. Uma subamostra de $100 \mathrm{mg}$ foi digerida em ácido sulfúrico concentrado e água oxigenada 30\% (100 volumes). As leituras dos macronutrientes ( $\mathrm{Ca}, \mathrm{Mg}$ e $\mathrm{K})$ e a do $\mathrm{Al}$ foram realizadas em espectrofotômetro de absorção atômica. $\mathrm{O}$ cálcio foi determinado por emissão, e o K, Mg e alumínio por absorção. O fósforo foi determinado por método colorimétrico usando o reagente molibdatovanadato de amônio, a $410 \mathrm{~nm}$ (Allen 1974).

\section{Resultados e Discussão}

Nas duas matas os teores dos macronutrientes analisados e do $\mathrm{Al}$ apresentaram o mesmo comportamento em cada categoria foliar. Durante o período chuvoso (Tabelas 1 e 2 ) os teores de $\mathrm{P}$ e $\mathrm{K}$ foram significativamente maiores (teste $\mathrm{t}, \mathrm{p}<0,05$ ) nas folhas novas, enquanto que o $\mathrm{Ca}, \mathrm{Mg}$ e $\mathrm{Al}$ ocorreram com maiores teores em folhas maduras e senescentes (teste $t, p<0,05$ ). Du- 
rante a seca (Tabelas 2 e 4), período que $M$. pubescens não produz folhas novas, as folhas maduras apresentaram maior teor de $\mathrm{P}$, enquanto que as senescentes tiveram maiores teores de $\mathrm{Ca}, \mathrm{Mg}$ e $\mathrm{Al}$. Para o $\mathrm{K}$ não houve diferença estatística entre estas duas categorias foliares. A ocorrência da maior concentração de $\mathrm{K}$ nas folhas novas deve estar relacionado com a mobilidade deste elemento, que é facilmente lixiviado ou translocado (Larcher 1975). Já o Ca e $\mathrm{Mg}$, considerados elementos pouco móveis, são em geral encontrados em maior concentração nas folhas maduras e senescentes (Larcher 1975).

A concentração média de alumínio nas folhas Metrodoreà pubescens variou de $308 \mathrm{ppm}$ (indivíduos com $5-50 \mathrm{~cm}$ ) a $1816 \mathrm{ppm}$ (indivíduos com $100-150 \mathrm{~cm})$. Variando também de folhas novas para folhas maduras. A concentração deste elemento em folhas novas foi sempre inferior a $1000 \mathrm{ppm}$, o mesmo não ocorreu para as folhas maduras. Uma espécie pode ser considerada acumuladora de alumínio se apresentar uma concentração deste elemento superior a $1000 \mathrm{ppm}$ (Haridasan 1982). Portanto, M. pubescens só poderia ser considerada acumuladora de alumínio se fossem consideradas apenas as folhas maduras e senescentes dos indivíduos com tamanho superior a $50 \mathrm{~cm}$. Assim, o estágio de desenvolvimento da planta e da folha parecem ser pontos importantes à serem considerados na determinação de uma espécie acumuladora de alumínio. Machado (1985) também observou uma menor acumulação de alumínio em indivíduos jovens de Vochysia thyrsoidea.

As maiores concentrações de $\mathrm{Ca}$ e de $\mathrm{K}$ nas folhas ocorreram no período seco, nas duas matas (teste $\mathrm{t},<0,01$ ). Para o $\mathrm{Al}$ não foi observado diferença significativa entre os períodos amostrados (teste $\mathrm{t},<0,05$ ). Já o $\mathrm{P}$ e o $\mathrm{Mg}$ apresentaram padrões diferentes entre as duas matas. $\mathrm{O} P$ não apresentou diferença entre os períodos amostrados na mata de galeria $(\mathrm{p}>0,05)$, porém sua concentração foi maior no período chuvoso na mata mesofítica (teste t, $\mathrm{p}<0,01)$. A maior concentração de $\mathrm{Mg}$ nas folhas ocorreu no período seco para a mata de galeria (teste $t, p<0,01$ ), não tendo sido observado diferença entre os períodos amostrados na mata mesofítica (teste t, $\mathrm{p}>0,05$ ).

Os indivíduos de $M$. pubescens da mata mesofítica (solo calcáreo) apresentaram maiores teores de $\mathrm{Ca}(\mathrm{t}=5,60, \mathrm{gl}=56, \mathrm{p}<0,01)$ e menores teores de $\mathrm{K}(\mathrm{t}=2,13, \mathrm{gl}=56, \mathrm{p}<0,05)$ do que as plantas na mata de galeria (solo ácido) nos dois períodos amostrados. Em geral, não ocorreram diferenças estatísticas em relação aos demais elementos analisados, com excessão do $\mathrm{Mg}$, que foi maior na mata mesofítica no período seco $\mathrm{gl}=14, \mathrm{p}<0,01$ ). Alguns autores têm verificado que populações de solos calcáreos e de solos ácidos podem diferir na habilidade de acumular diferentes nutrientes (Davies e Snaydon 1973, Wacquant e Boub 1983, Haridasan e Araújo 1988). Davies e Snaydon (1983) estudando uma população de Dittrichia viscosa em solo calcáreo e outra em solo ácido encontraram também o mesmo comportamento por nós observado em $M$. pubescens em relação a concentração de Ca e K. Estes autores sugerem que além das diferenças no solo, outros fatores como o comportamento fi- 
siológico das plantas devem ser considerados. Assim, as diferenças encontradas entre as populações de $M$. pubescens talvez possam ser explicadas simplesmente por diferenças de solo, entretanto as plantas desta espécie em solo ácido podem possuir um comportamento calcífuga e as do solo calcáreo um comportamento calcícola. Estudos mais detalhados de experimentação são necessários para elucidar esta questão.

Tabela 1 - Teores médios de $\mathrm{P}, \mathrm{K}, \mathrm{Ca}, \mathrm{Mg}(\%)$ e $\mathrm{Al}$ (ppm) em folhas novas (Fn), maduras (Fm) e senescentes (Fs) de Metrodorea pubescens na mata de galeria, durante o período chuvoso. O desvio padrão está entre parenteses. $n=5$.

\begin{tabular}{lccccc}
\hline $\begin{array}{l}\text { Estágio } \\
\text { foliar }\end{array}$ & $\mathrm{P}$ & $\mathrm{K}$ & $\mathrm{Ca}$ & $\mathrm{Mg}$ & $\mathrm{Al}$ \\
\hline \multirow{5}{*}{ Fn } & 0,32 & 2,45 & 0,46 & 0,36 & 320 \\
& $(0,03)$ & $(0,12)$ & $(0,26)$ & $(0,05)$ & $(85)$ \\
Fs & 0,22 & 2,30 & 0,78 & 0,44 & 665 \\
& $(0,01$ & $(0,28)$ & $(0,35)$ & $(0,05)$ & $(100)$ \\
& & $50-100 \mathrm{~cm}$ & & & \\
Fn & 0,32 & 2,63 & 0,22 & 0,32 & 720 \\
& $(0,06)$ & $(0,23)$ & $(0,03)$ & $(0,02)$ & $(230)$ \\
Fm & 0,26 & 2,43 & 0,041 & 0,36 & 1130 \\
& $(0,11)$ & $(0,06$ & $(0,11)$ & 0,40 & $(40)$ \\
Fs & 0,23 & 2,24 & 0,41 & $(0,02)$ & 1090 \\
& $(0,01)$ & $(0,06)$ & $(0,06)$ & & $(160)$ \\
& & & & & \\
& & $100-150 \mathrm{~cm}$ & & & \\
Fn & 0,34 & 2,69 & 0,29 & 0,33 & 945 \\
& $(0,05)$ & $(0,23)$ & $(0,06)$ & $(0,02)$ & $(60)$ \\
Fm & 0,25 & 2,41 & 0,54 & 0,39 & 1415 \\
& $(0,05)$ & $(0,07)$ & $(0,14)$ & $(0,03)$ & $(55)$ \\
Fs & 0,23 & 2,22 & 0,51 & 0,43 & 1258 \\
& $(0,10)$ & $(0,14)$ & $(0,09)$ & $(0,01)$ & $(80)$ \\
\hline
\end{tabular}


Tabela 2 - Teores médios de $\mathrm{P}, \mathrm{K}, \mathrm{Ca}, \mathrm{Mg}(\%)$ e $\mathrm{Al}(\mathrm{ppm})$ em folhas novas (Fn), maduras $(\mathrm{Fm})$ e senescentes (Fs) de Metrodorea pubescens na mata mesofítica, durante o período chovoso. O desvio padrão está entre parênteses. $n=5$.

\begin{tabular}{lccccc}
\hline $\begin{array}{l}\text { Estágio } \\
\text { foliar }\end{array}$ & $\mathrm{P}$ & $\mathrm{K}$ & $\mathrm{Ca}$ & $\mathrm{Mg}$ & $\mathrm{Al}$ \\
\hline \multirow{4}{*}{ Fn } & \multicolumn{2}{c}{$5-50 \mathrm{~cm}$} & & & \\
& 0,35 & 2,59 & 0,81 & 0,42 & 308 \\
Fs & $(0,09)$ & $(0,34)$ & $(0,17)$ & $(0,04)$ & $(55)$ \\
& 0,24 & 1,52 & 1,55 & 0,54 & 600 \\
& $(0,02)$ & $(0,14)$ & $(0,26)$ & $(0,03)$ & $(140)$ \\
Fn & 0,34 & 2,50 & 0,81 & 0,41 & 610 \\
& $(0,06)$ & $(0,26)$ & $(0,17)$ & $(0,03)$ & $(35)$ \\
Fm & 0,26 & 2,11 & 1,43 & 0,50 & 1130 \\
& $(0,02)$ & $(0,26)$ & $(0,14)$ & $(0,01)$ & $(40)$ \\
Fs & 0,23 & 1,64 & 1,77 & 0,55 & 885 \\
& $(0,01)$ & $(0,22)$ & $(0,19)$ & $(0,02)$ & $(60)$ \\
& & & & & \\
& 0,35 & 2,67 & 0,84 & 0,39 & 945 \\
Fn & $(0,04)$ & $(0,12)$ & $(0,19)$ & $(0,05)$ & $(65)$ \\
& 0,26 & 1,86 & 1,42 & 0,50 & 1250 \\
Fm & $(0,02)$ & $(0,41)$ & $(0,28)$ & $(0,05)$ & $(40)$ \\
& 0,23 & 1,72 & 1,55 & 0,53 & 1207 \\
Fs & $(0,03)$ & $(0,39)$ & $(0,38)$ & $(0,04)$ & $(30)$ \\
& & & &
\end{tabular}

Tabela 3 - Teores médios de $\mathrm{P}, \mathrm{K}, \mathrm{Ca}, \mathrm{Mg}(\%)$ e $\mathrm{Al}(\mathrm{ppm})$ em folhas maduras (Fm) e senescentes (Fs) de Metrodorea pubescens na mata de galeria, durante o período seco. O desvio padrão está entre parenteses. $n=5$.

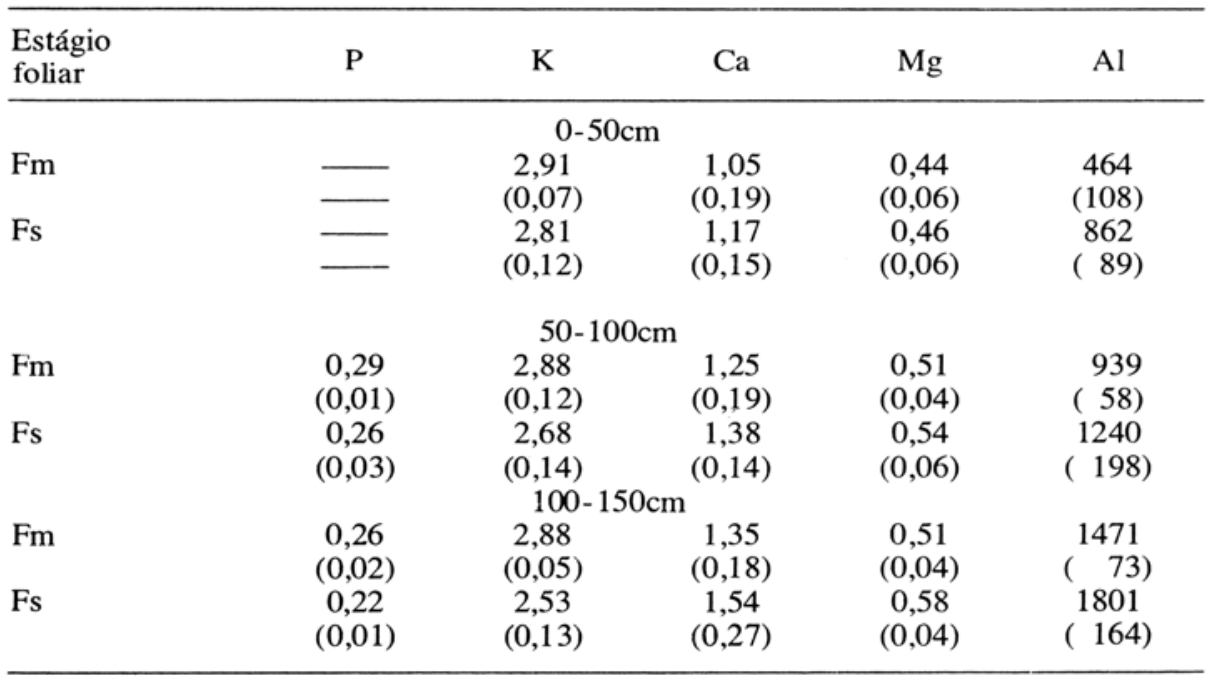


Tabela 4 - Teores médios de $\mathrm{P}, \mathrm{K}, \mathrm{Ca}, \mathrm{Mg}(\%)$ e $\mathrm{Al}(\mathrm{ppm})$ em folhas maduras (Fm) e senescentes (Fs) de Metrodorea pubescens na mata mesofítica, durante o período seco. $\mathrm{O}$ desvio padrão está entre parênteses. $\mathrm{n}=5$.

\begin{tabular}{|c|c|c|c|c|c|}
\hline $\begin{array}{l}\text { Estágio } \\
\text { foliar }\end{array}$ & $\mathrm{P}$ & $\mathrm{K}$ & $\mathrm{Ca}$ & $\mathrm{Mg}$ & $\mathrm{Al}$ \\
\hline \multicolumn{6}{|c|}{$0-50 \mathrm{~cm}$} \\
\hline $\mathrm{Fm}$ & $\begin{array}{c}0,23 \\
(0,02)\end{array}$ & $\begin{array}{c}2,78 \\
(0,09)\end{array}$ & $\begin{array}{c}1,67 \\
(0,15)\end{array}$ & $\begin{array}{c}0,48 \\
(0,03)\end{array}$ & $\begin{array}{c}344 \\
(55)\end{array}$ \\
\hline Fs & $\begin{array}{c}0,20 \\
(0,03)\end{array}$ & $\begin{array}{c}2,60 \\
(0,27)\end{array}$ & $\begin{array}{c}1,80 \\
(0,06)\end{array}$ & $\begin{array}{c}0,50 \\
(0,08)\end{array}$ & $\begin{array}{r}846 \\
(171)\end{array}$ \\
\hline \multicolumn{6}{|c|}{$50-100 \mathrm{~cm}$} \\
\hline $\mathrm{Fm}$ & $\begin{array}{c}0,21 \\
(0,01)\end{array}$ & $\begin{array}{c}2,79 \\
(0,14)\end{array}$ & $\begin{array}{c}1,67 \\
(0,25)\end{array}$ & $\begin{array}{c}0,48 \\
(0,04)\end{array}$ & $\begin{array}{r}898 \\
(65)\end{array}$ \\
\hline Fs & $\begin{array}{c}0,19 \\
(0,01)\end{array}$ & $\begin{array}{c}2,53 \\
(0,17) \\
50-1\end{array}$ & $\begin{array}{c}1,95 \\
(0,27)\end{array}$ & $\begin{array}{c}0,52 \\
(0,06)\end{array}$ & $\begin{array}{l}1189 \\
(126)\end{array}$ \\
\hline $\mathrm{Fm}$ & $\begin{array}{c}0,21 \\
(0,01)\end{array}$ & $\begin{array}{c}2,79 \\
(0,14)\end{array}$ & $\begin{array}{c}1,67 \\
(0,25)\end{array}$ & $\begin{array}{c}0,48 \\
(0,04)\end{array}$ & $\begin{array}{r}898 \\
(65)\end{array}$ \\
\hline Fs & $\begin{array}{c}0,19 \\
(0,01)\end{array}$ & $\begin{array}{c}2,53 \\
(0,17)\end{array}$ & $\begin{array}{c}1,95 \\
(0,27)\end{array}$ & $\begin{array}{c}0,52 \\
(0,06)\end{array}$ & $\begin{array}{l}1189 \\
(126)\end{array}$ \\
\hline $\mathrm{Fm}$ & $\begin{array}{c}0,23 \\
(0,01)\end{array}$ & $\begin{array}{c}100- \\
2,73 \\
(0,13)\end{array}$ & $\begin{array}{c}1,78 \\
(0,21)\end{array}$ & $\begin{array}{c}0,46 \\
(0,05)\end{array}$ & $\begin{array}{l}1391 \\
(187)\end{array}$ \\
\hline Fs & $\begin{array}{c}0,21 \\
(0,02)\end{array}$ & $\begin{array}{c}2,70 \\
(0,15)\end{array}$ & $\begin{array}{c}1,98 \\
(0,16)\end{array}$ & $\begin{array}{c}0,50 \\
(0,03)\end{array}$ & $\begin{array}{l}1816 \\
\text { ( } 57)\end{array}$ \\
\hline
\end{tabular}

\section{Agradecimentos}

Os autores gostariam de agradecer ao CNPq pela concessão de auxílio de pesquisa ao J.D.H. (Proc. 403.421/88-8) e a bolsa de mestrado ao M.T.N. A técnica Mara R. dos Santos pelo auxílio nas análises químicas e ao técnico Mardocheu Rocha pelo auxílio nas coletas de campo. Ao Prof. M. Haridasan por possibilitar o uso do laboratório de solos do Departamento de Ecologia na UnB e a Ms. Dora Maria Villela pelas críticas ao manuscrito.

\section{Referências Bibliográficas}

ALLEN, S.E. 1974. Chemical Analysis of Ecological Materials. Blackwell Scientific, Oxford.

DAVIES, M.S. \& R.W.SNAYDON. 1973. Phisiological differences among populations of Anthoxanthum odoratum L. collected from the Park Grass experiment, Rothamsted. J. Appl. Ecol. 10:47-58. 
EITEN, G. 1984. Vegetation of Brasilia. Phytocoenologia 12(2/3): 271-292. HARIDASAN, M. 1982. Aluminium accumulation by some cerrado native species of central Brazil. Plant \& Soil 65:265-273.

HARIDASAN, M. 1985. Accumulation of nutrients by eucalyptus seedlings from acidic and calcareous soils of the cerrado region of Central Brazil. Plant \& Soil 86:35-45.

HARIDASAN, M. \& G.M. ARAÚJO. 1988. Aluminium-Accumulating species in two forest communities in the cerrado region of central Brazil. Forest Ecology and Management 24:15-26.

LARCHER, W. 1975. Physiological Plant Ecology. Spring-Verlag, Berlin.

MACHADO, J.W.B. 1985. Acumulação de alumínio em Vochysia thyrsoidea Pohl. Dissertação de Mestrado, Departamento de Biologia Vegetal, Universidade de Brasília, Brazil.

NASCIMENTO, M.T. 1989. Herbivoria foliar em jovens de Metrodorea pubescens St. Hil \& Tull. (Rutaceae) em dois tipos de mata no Distrito Federal, Brasil. Dissertação de Mestrado, Departamento de Ecologia, Universidade de Brasília, Brasília-DF.

RAMOS, P.C. 1989. Estudos fitossociológicos em uma floresta mesofítica semidecídua na Fercal, Brasília-DF. Dissertação de Mestrado, Departamento de Biologia Vegetal, Universidade de Brasília, Brasília-DF.

WACQUANT, J.P. \& BOUAB, N. 1983. Nutritional differentiation within the species Dittrichia viscosa W. Greuter, between e population from a calcareous habitat and another from an acidic habitat. Plant \& Soil 72:297-303. 